\title{
SERTRALINE INDUCED OROMANDIBULAR DYSTONIA AND GALACTORRHEA: A CASE REPORT
}

\author{
Nilufer Doruk $^{1}$ \& Bekir Nihat Dogrul ${ }^{2}$ \\ ${ }^{1}$ Ankara University, Faculty of Medicine, Ankara, Turkey \\ ${ }^{2}$ University of Health Sciences, Gulhane Medical Faculty, Ankara, Turkey \\ received: 14.4.2021; \\ revised: 27.5.2021; \\ accepted: 1.6 .2021
}

\section{INTRODUCTION}

Selective Serotonin Reuptake Inhibitors (SSRI) are used frequently not only for their treatment efficacy, but also they are considered more tolerable and safe than other drugs used for anxiety and depression. However, studies and case reports have shown that extrapyramidal symptoms (EPS), which generally occur due to the use of antipsychotic drugs, also appear because of SSRI use. EPS induced by SSRIs appear in approximately 1 in 1000 or less (Sonmez \& Kosger 2015). The most common EPS associated with SSRI use are akathisia, dystonia, parkinsonism and dyskinesia. Although nonpuerperal lactation or galactorrhea is a common side effect attributable to the use of antipsychotic drugs, it is also encountered with SSRI use (Suthar et al. 2018). However, it is essential to pay attention to the rare side effects of commonly used drugs such as SSRIs.

In this case report, we present a case of oromandibular dystonia and galactorrhea due to sertraline use in a young female patient.

\section{CASE REPORT}

A 24-year-old single female who is a university student was admitted to the psychiatric outpatient clinic with complaints of anxiety related to daily life events, difficulty in falling asleep and maintaining sleep, palpitations, trembling in the hands, hot flushes, and uncontrollable crying spells. The patient stated that her complaints increase when she is alone. It was revealed that her complaints were mild and tolerable in the last few years but the severity of the symptoms increased in the last week.

There was not any psychiatric treatments in the medical history of the patient and physical examination findings were normal. The patient had no history of smoking, alcohol or substance abuse. She also did not have any diagnosed systemic diseases. She had mild iron-deficiency anemia. Except from low ferritin, the patient's complete blood count, chemistry panel and thyroid panel were normal. In the family history, her brother was diagnosed with major depression which was treated with antidepressant drugs.

In the psychiatric evaluation, the patient was diagnosed with F.41.2 mixed anxiety and depressive disorder according to the ICD diagnosis system. Sertraline $50 \mathrm{mg} /$ day was started for the treatment. The patient tolerated the drug well and there were no adverse effects. Significant improvements in symptoms were observed. However, in the fifth month of the treatment, the patient started to have tongue spasms with pain. Assuming this situation resulted from the drug, the dose of Sertraline was reduced to $25 \mathrm{mg}$ /day and this continued for 2 months. Although the complaints of tongue spasms and pain improved, dose of sertraline was increased to $50 \mathrm{mg} /$ day because of the rise in anxiety complaints. After 1 month, her anxiety complaints eased, but the spasms and pain in the tongue relapsed more severely than before. In addition to this symptoms, complaints of jaw spasms with pain and unilateral milk secretion began. There were not any pathological findings in physical examination, complete blood count and chemistry panel that could explain these complaints. During this period, the patient's menstruation cycles were regular like before. The patient, whose organic pathologies of the breast were excluded and the general system examination was within normal limits, was diagnosed with Sertralineinduced oromandibular dystonia and galactorrhea. The dose of sertraline was reduced gradually and discontinued. It was replaced with escitalopram $5 \mathrm{mg} /$ day. The patient's complaints cleared up completely on the third day. The treatment was continued with escitalopram $10 \mathrm{mg} /$ day. The patient is now in the fifth month of the escitalopram treatment, and the anxiety symptoms are in remission without drug-related complaints. When the patient started using Sertraline $50 \mathrm{mg} /$ day for the second time, Naranjo Adverse Drug Reaction Probability Scale (NADPRS) score was 9 related to dystonia symptoms (Naranjo et al. 1981). The patient did not use any other medication or nutritional supplements during this entire process. Written consent of the patient was obtained for this scientific study.

\section{DISCUSSION}

According to a study conducted by the Centers for Disease Control and Prevention, approximately 13 out of every 100 people in the adult age group used antidepressant drugs between the years 2015 and 2018 in the USA (Brody \& Gu 2020). Since these drugs are used so frequently, even it is rare, various side effects 
can be seen. In this case, resolving of symptoms after discontinuation of the drug and the Naranjo probability score of 9 (score of 9 and above considered definite association) suggests relationship between adverse effects and sertraline use. To our knowledge, this is the first case report in the literature in that sertraline-induced oromandibular dystonia and galactorrhea are observed together. In the management of dystonia, the dose of the causative drug can be reduced or drug can be replaced with an alternative. If necessary, antimuscarinic medications such as benztropine or trihexyphenidyl can be used. In this case, at the first time adverse effects appeared, the dose of the drug (sertraline) was reduced and the side effects were resolved. When the dose was increased because of the rise in anxiety complaints, the side effects appeared more severe and complex (galactorrhea in addition to EPS) than before. This time, the treatment was changed with another SSRI (escitalopram) and no side effects were observed afterwards. Both the response to the treatment and the drug tolerability were better.

The mechanism of EPS induced by SSRIs such as dystonia is not clearly elucidated, but it has been suggested that the inhibitory effect of serotonin on dopaminergic activity in extrapyramidal pathways or overstimulation of 5-HT2 receptors in the basal ganglia may cause such side effects (Zádori et al. 2015, Caffrey et al. 2020). Also, genetic mechanisms such as serotonin or dopamine receptor polymorphisms may play a role in the emergence of SSRI-induced EPS (Hedenmalm et al. 2006). SSRI induced galactorrhea can be associated with inhibition of dopamine activity at tuberoinfundibular pathway by serotonergic activation or direct stimulation of hypothalamic serotonergic receptors at paraventricular nucleus of hypothalamus (Chatterjee et al. 2015). It should be bear in mind that serum prolactin levels may not be high in cases of SSRI-induced galactorrhea (Halder et al. 2015).

In this case, observation of EPS after the patient's use of sertraline for 5 months indicates that such side effects may also occur in the later period. Although the majority of SSRI-induced EPS in the literature appear within the first 30 days after treatment initiation or dose increase, the onset of symptoms may extend up to the 18 months of treatment, which is consistent with our case (Hawthorne \& Caley 2015).

As a result, SSRIs can cause adverse effects such as EPS and galactorrhea. These adverse effects may show up in the late period of drug use. Changing the treatment with another SSRI is a good option for the management of adverse effects.

Acknowledgments: None.

Conflict of interest: None to declare.

\section{Contribution of individual authors:}

Nilufer Doruk \& Bekir Nihat Dogrul: made concept and design of the study, literature search and manuscript writing.

\section{References}

1. Brody DJ \& Gu Q: Antidepressant use among adults: United States, 2015-2018. NCHS Data Brief 2020; 377:1-8

2. Caffrey D, Sowden G \& Arsan C: A Possible Case of Escitalopram-Induced Tardive Dystonia. Psychosomatics 2020; 61:188-192

3. Chatterjee SS, Mitra $S \&$ Mallik N: Emerging Hyperprolactinemic Galactorrhea in Obsessive Compulsive Disorder with a Stable Dose of Fluoxetine. Clin Psychopharmacol Neurosci 2015; 13:316-318

4. Halder A, Saha P, Mandal U \& Biswas A: Selective serotonin reuptake inhibitors-paroxetine induced galactorrhea in a case of somatoform disorder. Indian $j$ health sci 2015; 8:64

5. Hawthorne JM \& Caley CF: Extrapyramidal Reactions Associated With Serotonergic Antidepressants. Ann Pharmacother 2015; 49:1136-1152

6. Hedenmalm K, Güzey C, Dahl M-L, Yue Q-Y \& Spigset O: Risk Factors for Extrapyramidal Symptoms During Treatment With Selective Serotonin Reuptake Inhibitors, Including Cytochrome P-450 Enzyme, and Serotonin and Dopamine Transporter and Receptor Polymorphisms. Journal of Clinical Psychopharmacology 2006; 26:192-197

7. Naranjo CA, Busto U, Sellers EM, Sandor P, Ruiz I, Roberts EA et al.: A method for estimating the probability of adverse drug reactions. Clin Pharmacol Ther 1981; 30:239-245

8. Sonmez I \& Kosger F: Venlafaxine-induced acute dystonia: a case report. Dusunen Adam 2015; 374-377

9. Suthar N, Pareek V, Nebhinani $N \&$ Suman D: Galactorrhea with antidepressants: $A$ case series. Indian $J$ Psychiatry 2018; 60:145

10. Zádori D, Veres $G$, Szalárdy L, Klivényi $P$ \& Vécsei L: Drug-induced movement disorders. Expert Opinion on Drug Safety 2015; 14:877-890
Correspondence:

Bekir Nihat Dogrul, MD

University of Health Sciences, Gulhane Medical Faculty

Emrah, Gulhane Street, 06010 Keçioren, Ankara, Turkey

E-mail:nihatdogrul@outlook.com 